\title{
Incorporating magnetic field observations in wind models of low-mass stars
}

\author{
A. A. Vidotto ${ }^{1,2}$ \\ ${ }^{1}$ School of Physics \& Astronomy, Univ. of St Andrews, North Haugh, St Andrews, KY16 9SS, UK \\ ${ }^{2}$ Observatoire de Genève, Univ. de Genève, Ch. des Maillettes 51, Versoix, 1290, Switzerland \\ Correspondence to: A. A. Vidotto (aline.vidotto@unige.ch)
}

Received: 24 March 2014 - Revised: 21 May 2014 - Accepted: 22 May 2014 - Published: 6 June 2014

\begin{abstract}
Stellar winds of cool, main-sequence stars are very tenuous and difficult to observe. Despite carrying away only a small amount of the stellar mass, they are important for regulating the rotation of the star and, consequently, its activity and magnetism. As it permeates the interplanetary space, the stellar wind interacts with any exoplanet encountered on its way, until it reaches the interstellar medium (ISM). These interactions can result in complex physical processes that depend on the characteristics of the wind. To better constrain the wind characteristics, more realistic wind models that account for factors such as stellar rotation and the complex/diverse observationally-derived stellar magnetic field configurations of cool stars are required. In this paper, I present a three-dimensional model of the wind of cool stars, which adopt as boundary condition observationally-derived magnetic maps. I also discuss how these studies are relevant for, e.g., the characterisation of the interaction between stellar winds and planets/ISM, and the propagation of cosmic rays.
\end{abstract}

\section{Introduction}

Magnetic fields play an important role along the stellar life. For low-mass stars, they are believed to regulate stellar rotation from the early stages of star formation until the ultimate stages of the life of a star. Empirically, the projected rotational velocities $v \sin (i)$ of G-type stars in the main-sequence phase decrease with age $t$ as $v \sin (i) \propto t^{-1 / 2}$ (Skumanich, 1972). This rotational braking is believed to be caused by stellar winds, which, outflowing along magnetic field lines, are able to efficiently remove the angular momentum of the star (e.g., Parker, 1958; Weber and Davis Jr., 1967).

As the wind flows out of the star, it impinges on any exoplanet encountered on its way, all the way until it reaches the ISM. The interactions between the stellar wind and exoplanets/ISM can result in complex physical processes that depend on the characteristics of the wind. These characteristics depend, among others, on the particular geometry of the stellar magnetic field. Modern techniques, such as ZeemanDoppler Imaging (ZDI), have made it possible to reconstruct the large-scale surface magnetic fields of other stars (Donati and Landstreet, 2009). This method has now been used to investigate the magnetic topology (i.e., intensity and orientation) of more than 100 low-mass stars. Thanks to this intense observational effort, a more general understanding of how the large-scale field correlates with age, activity and rotation is starting to emerge (Vidotto et al., 2014a). In addition, given all this recent insight gained into the magnetic topology of different stars, we are now able to produce more realistic models of winds of low-mass stars, directly incorporating the observationally derived stellar magnetism into three-dimensional (3-D) numerical simulations.

\section{Numerical models of stellar winds}

To account for the observed 3-D nature of stellar magnetic fields, 3-D stellar wind models are required. Here I present the technique used in Vidotto et al. (2011b, 2012, 2014b), Jardine et al. (2013), and Llama et al. (2013), which incorporates the observationally reconstructed magnetic fields using the ZDI technique. The wind models were simulated 
using the 3-D magnetohydrodynamics (MHD) numerical code BATS-R-US (Powell et al., 1999). BATS-R-US solves the ideal MHD equations

$$
\begin{aligned}
& \frac{\partial \rho}{\partial t}+\nabla \cdot(\rho \boldsymbol{u})=0 \\
& \frac{\partial(\rho \boldsymbol{u})}{\partial t}+\nabla \cdot\left[\rho \boldsymbol{u} \boldsymbol{u}+\left(P+\frac{B^{2}}{8 \pi}\right) I-\frac{\boldsymbol{B} \boldsymbol{B}}{4 \pi}\right]=\rho \boldsymbol{g} \\
& \frac{\partial \boldsymbol{B}}{\partial t}+\nabla \cdot(\boldsymbol{u} \boldsymbol{B}-\boldsymbol{B} \boldsymbol{u})=0 \\
& \frac{\partial}{\partial t}\left[\frac{\rho u^{2}}{2}+\frac{P}{\gamma-1}+\frac{B^{2}}{8 \pi}\right]+\nabla \cdot\left\{\boldsymbol { u } \left[\left(\frac{\rho u^{2}}{2}+\frac{P}{\gamma-1}+\frac{B^{2}}{8 \pi}\right)\right.\right. \\
& \left.\left.\quad+P+\frac{B^{2}}{8 \pi}\right]-\frac{(\boldsymbol{u} \cdot \boldsymbol{B}) \boldsymbol{B}}{4 \pi}\right\}=\rho \boldsymbol{g} \cdot \boldsymbol{u}
\end{aligned}
$$

at the inertial reference frame, where the eight primary variables are the mass density $\rho$, the plasma velocity $\boldsymbol{u}$, the magnetic field $\boldsymbol{B}$, and the gas pressure $P$. The gravitational acceleration is given by $\boldsymbol{g}$, and $\gamma$ is the polytropic index $\left(p \propto \rho^{\gamma}\right)$. We consider an ideal gas, so $P=n k_{B} T$, where $k_{B}$ is the Boltzmann constant, $T$ is the temperature, $n=\rho /\left(\mu m_{p}\right)$ is the particle number density of the stellar wind, $\mu m_{p}$ is the mean mass of the particle.

Simulation grid: our grid is Cartesian and, for the simulations we illustrate next, the grid extends in $x, y$, and $z$ from -20 to 20 stellar radii $\left(R_{\star}\right)$. The star is placed at the centre of the grid. The finest resolved cells (resolution of $0.0097 R_{\star}$ ) are located close to the star and the coarsest cells $\left(0.31 R_{\star}\right)$ are located at the outer edges of the grid. The total number of cells in our simulations is $\sim 80$ million.

Boundary conditions: the inner boundary of our simulation is located at $r=R_{\star}$, where the radial component of the magnetic field $\left(B_{\mathrm{r}}\right)$ is set to be the observationallyreconstructed one. $B_{\mathrm{r}}$ is held fixed at the boundary throughout the simulation run, as are $\rho$ and $P$. A zero radial gradient is set to the remaining components of $\boldsymbol{B}$ and $\boldsymbol{u}=0$ in the frame co-rotating with the star. At the outer boundaries (at the edges of the grid), a zero gradient is set to all the primary variables. The rotation axis of the star is aligned with the $z$ axis, and the star is assumed to rotate as a solid body.

Initial state: at the initial state of the simulations, the wind profile is that of a non-magnetised, thermallydriven wind (Parker, 1958). The magnetic field considered in the grid is derived from extrapolations of observed surface radial magnetic maps using the potentialfield source surface method (Altschuler and Newkirk, 1969). Starting from an initial potential magnetic field configuration and a thermally-driven wind, the system is then evolved in time, resulting in a self-consistent interaction of the wind particles and the magnetic field lines.
The resultant solution, obtained self-consistently, is found when the system reaches steady state in the reference frame co-rotating with the star. The left (right) panel of Fig. 1 illustrates the initial (final) configuration of the magnetic field lines in the wind simulation of DT Vir, based on the simulations presented in Vidotto et al. (2014b). Note that, because of the interaction between particles and magnetic field lines, currents are created in the system and the magnetic field is removed from its initial potential configuration, becoming stressed. DT Vir is an early-M dwarf star, with a mass of $0.59 M_{\odot}$, radius $0.53 R_{\odot}$ and rotation period of $2.85 \mathrm{~d}$. We assume $\gamma=1.1$ and $\mu=0.5$. The other free parameters of our model are the wind base temperature $\left(2 \times 10^{6} \mathrm{~K}\right)$ and the density $\left(10^{11} \mathrm{~g} \mathrm{~cm}^{-3}\right)$. Ideally, we would like to constrain these parameters from observations of winds of cool stars, but these are rather challenging to obtain and no wind constraints exist for DT Vir. On the other hand, X-ray observations can provide estimates on the coronal temperatures and densities (Güdel, 2004), helping to constrain the densities and temperatures at the wind base. The values adopted for DT Vir are within the observed range provided by X-ray observations.

In total, Vidotto et al. (2014b) performed numerical simulations of the winds of a sample of 6 early-M dwarfs whose magnetic fields were observationally reconstructed (Donati et al., 2008). They showed that the wind mass flux is essentially modulated by the local value of $\left|B_{\mathrm{r}}\right|$, which share roughly the same modulation as the surface $\left|B_{\mathrm{r}}\right|$ (observed). This implies that the more non-axisymmetric topology of the stellar magnetic field results in more asymmetric mass fluxes.

\section{A few comments on the relevance of stellar wind studies}

The study of the local characteristics of the stellar wind is important for the characterisation of complex interactions that take place between exoplanets and their host stars. Planetary magnetospheric sizes are roughly set by pressure equilibrium between the planet's magnetic field and the stellar wind total pressure $p_{\text {tot }}$ (i.e., the sum of thermal, magnetic and ram pressures). Vidotto et al. (2014b) found that, close to the star, $p_{\text {tot }}$ is modulated by $\left|B_{\mathrm{r}}\right|$ and the more non-axisymmetric topology of the stellar magnetic field produces more asymmetric distributions of $p_{\text {tot }}$. As an exoplanet orbits around its host star, it probes regions of different $p_{\text {tot }}$. Consequently, its magnetospheric size becomes smaller (larger) when the external $p_{\text {tot }}$ is larger (smaller). In addition to magnetospheric size variations, the orientation of the bow shock that forms surrounding planetary magnetospheres also changes along the planetary orbit, as a consequence of the asymmetric nature of stellar magnetic fields. Other effects, such as planetary obliquity, eccentricity, and temporal variations of the stellar magnetic field, can also cause variability in planetary magnetospheric characteristics (Vidotto et al., 2011a). 

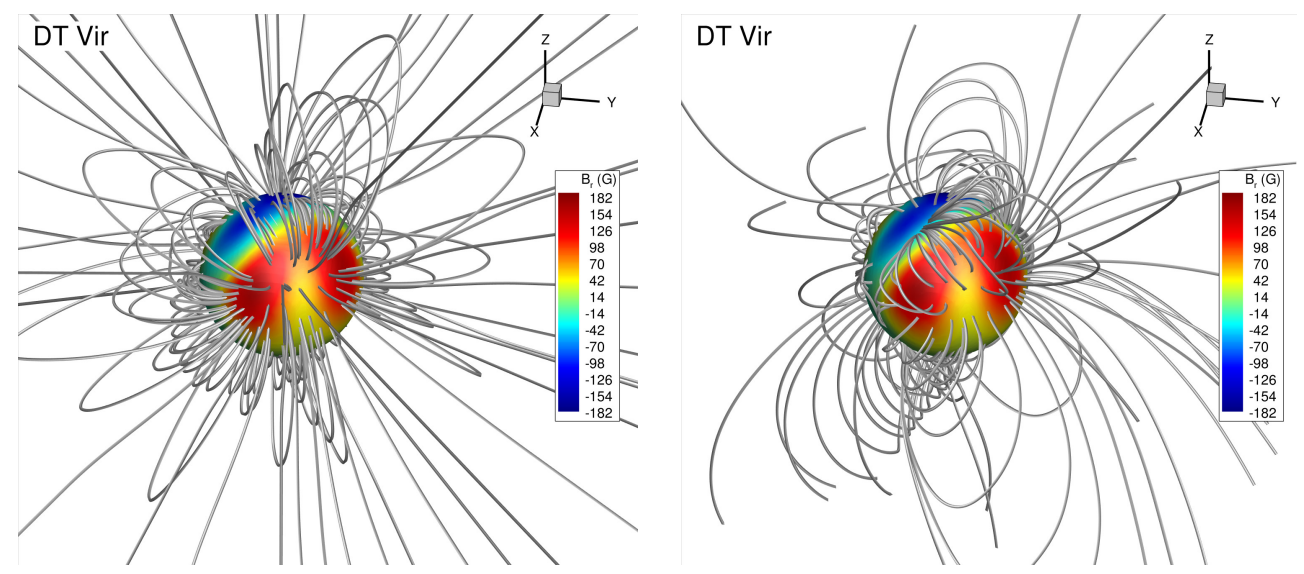

Figure 1. Left: the initial configuration of the magnetic field lines extrapolated from observed surface radial magnetic maps (colour-coded, from Donati et al., 2008) assuming a potential field. Right: final configuration of the magnetic field, derived from self-consistent interaction between wind particles and magnetic field lines.

The stellar wind can also affect the distances and shapes of "astropauses", defined as the surface where the pressure of the stellar wind balances the pressure of the ISM. In the presence of highly asymmetric stellar winds, which could be caused by the non-axisymmetric nature of the stellar magnetic field, astropauses might also lack symmetry. This can be a long-lasting phenomena or occur only at certain epochs of a magnetic cycle. Indeed, models of the interaction zone between the solar wind and the ISM suggests that the heliopause can be influenced due to solar cycle effects (Borovikov and Pogorelov, 2014). In the case of the solar system, the shape of the termination shock is likely more strongly influenced than the shape of the heliopause (e.g., Scherer and Fahr, 2003) and this might also be the case in stellar systems.

Finally, the geometry of $\boldsymbol{B}$ and the global characteristics of stellar winds can also affect propagation of cosmic rays (CRs). In the case of the Earth, the galactic CR flux is modulated over the solar cycle, being inversely correlated with the non-axisymmetric component of the total open solar magnetic flux (Wang et al., 2006). By analogy, if the nonaxisymmetric component of the stellar magnetic field is able to reduce the flux of CRs reaching an exoplanet, then we would expect that planets orbiting stars with largely nonaxisymmetric fields would be more shielded from galactic CRs, independently of the planet's own shielding mechanism (such as the ones provided by a thick atmosphere or large magnetosphere). The star can also be an important source of CRs. In the case of active stars, intensive fluxes of energetic electrons might remain trapped in the closed magnetic field structures. Close-in exoplanets that cross these large-scale structures might be exposed to intensive stellar CRs. In this case, the analogy regarding the non-axisymmetric component of B, which anti-correlates with the CRs flux on Earth, might not be valid. More study on that issue is needed.
Acknowledgements. I would like to thank the organisers for inviting me to attend this workshop and for contributing towards my travel expenses. I also thank the referees ( $\mathrm{T}$. Wiengarten and an anonymous one) who provided constructive feedbacks during the preparation of this manuscript. I acknowledge support from a Royal Astronomical Society Fellowship and an Ambizione Fellowship from the Swiss National Science Foundation.

Edited by: K. Scherer

Reviewed by: T. Wiengarten and one anonymous referee

\section{References}

Altschuler, M. D. and Newkirk, G.: Magnetic Fields and the Structure of the Solar Corona. I: Methods of Calculating Coronal Fields, Sol. Phys., 9, 131-149, 1969.

Borovikov, S. N. and Pogorelov, N. V.: Voyager 1 near the Heliopause, Astrophys. J., 783, L16, doi:10.1088/20418205/783/1/L16, 2014.

Donati, J.-F. and Landstreet, J. D.: Magnetic Fields of Nondegenerate Stars, Annu. Rev. Astron. Astr., 47, 333-370, doi:10.1146/annurev-astro-082708-101833, 2009

Donati, J.-F., Morin, J., Petit, P., Delfosse, X., Forveille, T., Aurière, M., Cabanac, R., Dintrans, B., Fares, R., Gastine, T., Jardine, M. M., Lignières, F., Paletou, F., Ramirez Velez, J. C., and Théado, S.: Large-scale magnetic topologies of early M dwarfs, Mon. Not. R. Astron. Soc., 390, 545-560, doi:10.1111/j.13652966.2008.13799.x, 2008.

Güdel, M.: X-ray astronomy of stellar coronae, Astron. Astrophys. Rev., 12, 71-237, 2004.

Jardine, M., Vidotto, A. A., van Ballegooijen, A., Donati, J.-F., Morin, J., Fares, R., and Gombosi, T. I.: Influence of surface stressing on stellar coronae and winds, Mon. Not. R. Astron. Soc., 431, 528-538, 2013.

Llama, J., Vidotto, A. A., Jardine, M., Wood, K., Fares, R., and Gombosi, T. I.: Exoplanet transit variability: bow shocks and winds around HD 189733b, Mon. Not. R. Astron. Soc., 436, 2179-2187, 2013. 
Parker, E. N.: Dynamics of the Interplanetary Gas and Magnetic Fields, Astrophys. J., 128, p. 664, doi:10.1086/146579, 1958.

Powell, K. G., Roe, P. L., Linde, T. J., Gombosi, T. I., and De Zeeuw, D. L.: A Solution-Adaptive Upwind Scheme for Ideal Magnetohydrodynamics, J. Comput. Phys., 154, 284-309, 1999.

Scherer, K. and Fahr, H. J.: Solar cycle induced variations of the outer heliospheric structures, Geophys. Res. Lett., 30, 1045, doi:10.1029/2002GL016073, 2003.

Skumanich, A.: Time Scales for CA II Emission Decay, Rotational Braking, and Lithium Depletion, Astrophys. J., 171, 565-567, 1972.

Vidotto, A. A., Jardine, M., and Helling, C.: Transit variability in bow shock-hosting planets, Mon. Not. R. Astron. Soc., 414, 1573-1582, 2011a.

Vidotto, A. A., Jardine, M., Opher, M., Donati, J. F., and Gombosi, T. I.: Powerful winds from low-mass stars: V374 Peg, Mon. Not. R. Astron. Soc., 412, 351-362, 2011 b.
Vidotto, A. A., Fares, R., Jardine, M., Donati, J.-F., Opher, M., Moutou, C., Catala, C., and Gombosi, T. I.: The stellar wind cycles and planetary radio emission of the $\tau$ Boo system, Mon. Not. R. Astron. Soc., 423, 3285-3298, 2012.

Vidotto, A. A., Gregory, S. G., Jardine, M., Donati, J.-F., Petit, P., Morin, J., Folsom, C. P., Bouvier, J., Cameron, A. C., Hussain, G., Marsden, S., Waite, I. A., Fares, R., Jeffers, S., and do Nascimento Jr, J. D.: Stellar magnetism: empirical trends with age and rotation, Mon. Not. R. Astron. Soc., 441, 2361-2374, 2014a.

Vidotto, A. A., Jardine, M., Morin, J., Donati, J. F., Opher, M., and Gombosi, T. I.: M-dwarf stellar winds: the effects of realistic magnetic geometry on rotational evolution and planets, Mon. Not. R. Astron. Soc., 438, 1162-1175, 2014b.

Wang, Y.-M., Sheeley Jr., N. R., and Rouillard, A. P.: Role of the Sun's Nonaxisymmetric Open Flux in Cosmic-Ray Modulation, Astrophys. J., 644, 638-645, 2006.

Weber, E. J. and Davis Jr., L.: The Angular Momentum of the Solar Wind, Astrophys. J., 148, 217-227, 1967. 